\title{
Nodes selection mechanism based on modified binary particle swarm optimization algorithm
}

\author{
Shengyun Wei ${ }^{1, a}$, Jing Zhang ${ }^{1, b}$, Taichuan Sun ${ }^{1, c}$ \\ ${ }^{1} \mathrm{PLA}$ Information Engineering University, China \\ ajunyun1002@126.com, b'zhangjingok@sina.com, 675551475@qq.com
}

Keywords: wireless sensor networks; node selection; binary particle swarm optimization; penalty function method

\begin{abstract}
Considering the problem of nodes selection in multi-target tracking of wireless sensor networks, a modified binary particle swarm optimization with a novel particle encoding method and particle position update rules and a mutation operation. Based on this modified binary particle swarm optimization, a nodes selection mechanism was proposed to maximize measurement information. The penalty function method was used to convert the constraint problem into an unconstrained one. The mechanism exploited the convergence speed and global search ability of the particle swarm optimization algorithm to make the optimization process achieving high-quality solutions in a short period of time. Finally, the experimental results show that the proposed nodes selection mechanism is significantly better than the classical branch and bound algorithm, and satisfies the needs of large-scale network nodes selection.
\end{abstract}

\section{Introduce}

Wireless sensor networks is a network system that integrated monitoring, control and wireless communication. It has broad application, such as military reconnaissance, environmental monitoring, target tracking, space exploration and disaster emergency. Especially, the network characteristics of rapid expansion, distributed information processing and anti-destroying ability, for maneuvering target tracking has created one of the most representative applications of WSN[1].

In recent years, many methods and strategies are proposed for the nodes selection problem. A mixed integer programming model established in [2], which using the branch and bound algorithm to solve the nodes selection problem. [3]took the diversity into account, partitioning the region based on the target prediction location, and selecting closest sensor to the target in each sector, then calculate the object function for each set to find the best active set. A global node selection algorithm was proposed in [4], which derives the boundaries of the target tracking performance, using a greedy strategy that "add one node at a time" and "simplify" for node selection. All the above are only for a single target, [5] considered the distributed node selection for multi-target tracking problem. The initial 0-1 integer programming problem is relaxed into a convex optimization problem, using gradient projection search as the solver.

In this paper, we consider the problem of nodes selection in multi-target tracking to maximize the measurement information. A modified discrete binary particle swarm optimization (MBPSO) algorithm with V-shaped transfer function is proposed as the solver. Finally, through multi-set of simulation experiment proves the feasibility and effectiveness of the algorithm.

\section{Modified binary particle swarm optimization, MBPSO}

In basic BPSO, along with the iterations, the random walk slows down the convergence speed of the algorithm. In addition, it is easy to fall into local optimum [6]. Therefore, a modified version of BPSO is proposed.

Velocity and position updating for MBPSO. In BPSO, a particle represents a possible solutions. The fitness of particles reflect their performance. Each particle has a D dimension position vector

$$
\mathbf{x}_{i}=\left(x_{i 1}, x_{i 2}, \ldots, x_{i D}\right) \quad \text { velocity vector }
$$$$
\mathbf{v}_{i}=\left(v_{i 1}, v_{i 2}, \ldots, v_{i D}\right) \text {, best position }
$$ 
$\mathbf{p}_{\text {best }, i}=\left(p_{\text {best }, i 1}, p_{\text {best }, i 2}, \ldots, p_{\text {best }, i D}\right)$, global best position of the population $\mathbf{G}_{\text {best }}=\left(\mathrm{g}_{\text {best }, 1}, \mathrm{~g}_{\text {best }, 2}, \ldots, \mathrm{g}_{\text {best }, \mathrm{D}}\right)$. The velocity of the particle is updated as:

$$
v_{i, d}^{t+1}=w \cdot v_{i, d}^{t}+c_{1} \cdot r_{1} \cdot\left(p_{\text {best }, i d}^{t}-x_{i, d}^{t}\right)+c_{2} \cdot r_{2} \cdot\left(\mathrm{g}_{\text {best }, d}^{t}-x_{i, d}^{t}\right)
$$

Where $v_{i, d}^{t}$ is the velocity of particle $i$ at iteration $t$ in the $d$ dimension. $c_{1}$ and $c_{2}$ are the acceleration coefficient. $r_{1}$ and $r_{2}$ are random numbers in the range of $[0,1] . w$ is the inertia weight.

Inertia weight plays an important role in balancing the global search and local search. This paper takes the inertia weight as:

$$
w=w_{\max }-\left(w_{\max }-w_{\min }\right)\left(\frac{t}{\text { Max_Iter }}\right)^{2}
$$

This article uses the V-shaped transfer function is described as:

$$
T\left(v_{i, d}^{t+1}\right)=\left|\operatorname{erf}\left(\frac{\sqrt{\pi}}{2}\right) v_{i, d}^{t+1}\right|=\left|\frac{\sqrt{2}}{\pi} \int_{0}^{(\sqrt{\pi} / 2) v_{i, d}^{t+1}} e^{-t^{2}} d t\right|
$$

The updating rules for positions based on the velocity corresponding (3) is:

$$
x_{i, d}^{t+1}= \begin{cases}0, & \text { if } \operatorname{rand}() \leq T\left(v_{i, d}^{t+1}\right) \\ 1, & \text { if } \operatorname{rand}()>T\left(v_{i, d}^{t+1}\right)\end{cases}
$$

Mutation operation for MBPSO. In order to solve the problem of local optimum. The mutation operation is introduced into MBPSO. When the evolution of the population is stagnation, and stagnation number reaches a certain threshold, the algorithm performs mutation operation. Mutation operation can increase the diversity of the population .The mutation operation is expressed as:

$$
x_{i, d}^{t}= \begin{cases}1-x_{i, d}^{t} & \text { if } \operatorname{rand}()<p_{m} \text { and } N>\lambda \\ x_{i, d}^{t} \quad \text { else }\end{cases}
$$

Where $N$ is the number of stagnation, $\lambda$ is the threshold, $p_{m}$ is the mutation rate.

\section{Problem formulation}

RSS based measurement model. RSS based measurement model uses attenuation characteristics of the signal communication to estimate the distance. This method does not require the time synchronization, and no extra hardware requirements, therefore, it is suitable for WSN[7]. The distance between node $i$ and target $k_{\text {is }} r_{i, k}=\left\|\mathbf{s}_{i}-\mathbf{p}_{k}\right\|$, where $\mathbf{s}_{i}=\left(s_{x, i}, s_{\mathrm{y}, i}\right)$ is the location of nodes $i$, $\mathbf{p}_{k}=\left(\mathrm{p}_{x, k}, \mathrm{p}_{y, k}\right)$ is the prediction location of target $k$, RSS based measurement model is expressed as:

$$
z_{i, k}(k)=P_{0}-10 \eta \log _{10}\left(r_{i, k} / r_{0}\right)+w_{i, k}(k)
$$

$P_{0}$ is the transmission power, $r_{0}=1$ is the reference distance. $\eta \in[2,5]$ is the path loss exponent, $w_{i, k}(k)$ is the Gaussian random variables, $w_{i, k}(k) \sim N\left(0, \sigma_{i, k}^{2}\right)$

Node selection optimization model. The key to improve the target tracking accuracy is related node accurately locate the target at each time step. Larger FIM indicates that more information can be obtained from the measurement, and gets a higher estimation accuracy.

The coordinates of the nodes are expressed in the form of polar coordinates, $\left(r_{i, k}, \phi_{i, k}\right)$, where $\phi_{i, k}$ is the angle between node $i$ and target $k, \cos \phi_{i, k}=\left(s_{x, i}-p_{x, k}\right) / r_{i, k}$ and $\sin \phi_{i, k}=\left(s_{y, i}-p_{y, k}\right) / r_{i, k}$,we can get the FIM:

$$
\mathbf{J}=\sum_{i \in \Psi_{t}^{m}} g_{i, k}\left[\begin{array}{cc}
\cos ^{2} \phi_{i, k} & \cos \phi_{i, k} \sin \phi_{i, k} \\
\cos \phi_{i, k} \sin \phi_{i, k} & \sin ^{2} \phi_{i, k}
\end{array}\right]
$$


Where, $\boldsymbol{\psi}_{t}^{m}$ is the active nodes set, $g_{i, k}$ is the function of $\sigma_{i, k}$ and $r_{i, k}$ :

$$
g_{i, k}=100 \eta^{2} /\left(\ln ^{2}(10) r_{i, k}^{2} \sigma_{i, k}^{2}\right)
$$

$X$ is defined as the nodes selection matrix, $N_{s}$ is the number of targets, $N_{a}$ is the number of nodes, Matrix element $X_{i, k}$ represents the relationship between the nodes and the targets

$$
x_{i, k}= \begin{cases}1 & \text { node } i \text { is selected for target } k \\ 0 & \text { else }\end{cases}
$$

Due to the FIM is a matrix form, which is not convenient to analysis. [8] has proved that the optimization problem of $\mathbf{J}$ and det $\mathbf{J}$ is equivalent, We can get the objective function of nodes selection as follows:

$$
f=\operatorname{det} \mathbf{J}=\frac{1}{2} \sum_{k=1}^{N_{s}} \sum_{i=1}^{N_{a}} \sum_{j=1}^{N_{a}} x_{i, k} \cdot x_{j, k} \cdot g_{i, k} \cdot g_{j, k} \cdot \sin ^{2}\left(\phi_{i, k}-\phi_{j, k}\right)
$$

Constraint 1 . One node can only track one target at a time:

$$
\sum_{k=1}^{N_{s}} x_{i, k} \leq 1 \quad i=1, \cdots N_{a}
$$

Constraint 2. The cardinal number of active set is $m$ :

$$
\sum_{i=1}^{N_{a}} x_{i, k}=m \quad k=1, \cdots N_{s}
$$

\begin{tabular}{|c|c|c|c|c|c|c|c|c|c|}
\hline Taxget 1 & Tareet2 & $\begin{array}{c}\text { Taxset } \\
\mathrm{Ni}\end{array}$ & $\begin{array}{c}\text { Taxset } \\
\text { Ns }\end{array}$ & & & Tareet1 & Tareet2 & $\begin{array}{c}\text { Taxget } \\
\mathrm{Ni}\end{array}$ & $\begin{array}{c}\text { Taxset } \\
\text { Ns }\end{array}$ \\
\hline 0 & 1 & $\ldots$ & $\mathbf{0}$ & $\cdots$ & $\ldots$ & 1 & 0 & $\cdots$ & $\mathbf{0}$ \\
\hline
\end{tabular}

\section{MBPSO based nodes selection algorithm}

Particle encoding. The main idea of this method is converting the matrix $X$ into a vector with $N_{s} \times N_{a}$ elements, so that each particle's position vector can represent the nodes selection result. Fig. 1 shows the structure of a particle.

Fig. 1 The structure of a particle

Fitness function. We use the static penalty function method to make the constrained problem into an unconstrained one. In addition, we solve the problem by translating $\max f$ into $\min -f$. So the fitness function is expressed as

$$
\text { fitness }(\mathbf{x}, \xi)=-f+\xi_{1} \cdot \sum_{i=1}^{N a}\left[\max \left\{0,\left(\sum_{k=1}^{N_{s}} a_{i, k}-1\right)\right\}^{2}+\xi_{2} \cdot \sum_{k=1}^{N s}\left(\sum_{i=1}^{N_{a}} a_{i, k}-m\right)^{2}\right.
$$

Where $\xi_{1}$ and $\xi_{2}$ are the punishment factor of inequality constraints and equality constraints.

Steps of Algorithm. Step1 Initialize the parameters for WSN and MBPSO.

Step2 Calculate the fitness. Calculate the fitness for each particles, obtain the best position $\mathbf{P}_{\text {best }, i}$ as the individual best, and the global best position $\mathbf{G}_{\text {best }}$ as the population best.

Step3 Update the particle's velocity and the binary position.

Step4 Judge the stagnation number and execute the mutation operation.

Step5 Calculate the fitness for the updated position, and update $\mathbf{P}_{\text {best }, i}$ and $\mathbf{G}_{\text {best }}$.

Step6 Algorithm terminate while the maximum number of iterations is reached and go to step 7. Otherwise, return to step 2.

Step7 Save $\mathbf{G}_{\text {best }}$ as the solution for the nodes selection problem. 


\section{Simulation Experiment and Result Analysis}

Simulation Environment Setting. In order to evaluate and analyze the performance of the proposed algorithm, we do a series of simulation experiments in the $2.0 \mathrm{GHz}$ PC by MATLAB. The parameters for WSN: the number of nodes is $N_{a} \in\{50,100\}$, they are randomly deployed in the monitoring area of $100 m \times 100 m, N_{s}=2, \eta=3$. The prediction position of targets for nodes selection get from filtering algorithm. The parameters for BPSO: $v_{\max }=6, c_{1}=c_{2}=2, \lambda=10$, Max_Iter $=500, w_{\max }=0.9, w_{\min }=0.4, p_{m}=0.02$, population size $n o P=30$, punishment factor $\xi_{1}=10, \xi_{2}=100$ 。

Simulation Results. In order to analysis the performance of MBPSO, which using V-shaped transfer function and mutation operation, we make it compare with the basic BPSO and the VBPSO with the V-shaped transfer function. Parameters setting: $N_{s}=2, N_{a}=50, m=3$. Experiments repeat 50 times for each algorithm. The simulation result shows in Fig.3. In the early iterations, the convergence speed of three algorithm are fast, however, the basic BPSO based nodes selection algorithm do not find the satisfied solution, and the VBPSO based nodes selection algorithm fall into local optimum. MBPSO algorithm not only obtain the high quality of feasible solution, and convergence speed faster, this is because the v-shaped MBPSO algorithm conversion function reduces the random walk of the particles in the process of iterations, improves the convergence speed. The mutation operation enables the algorithm to jump out of local optimum.

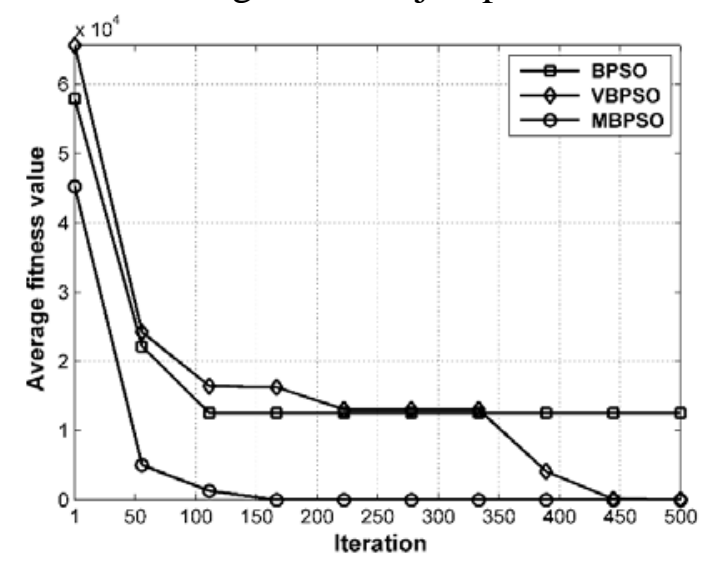

Fig.2 The performance of MBPSO based nodes selection algorithm

Fig.3 shows the performance with difference $m$. It is observed that, when $\mathrm{m}$ is small, the measurement information obtained by the network is small. When m increased from 4 to 7 , the fitness function value gets smaller, but not obviously. Therefore, it is important to choose a reasonable number of active nodes. The bigger $\mathrm{m}$ brings morn energy consumption. Therefore, comprehensive consider energy consumption, algorithmic complexity and performance requirements, $\mathrm{m}=3$ is employed for the MBPSO.
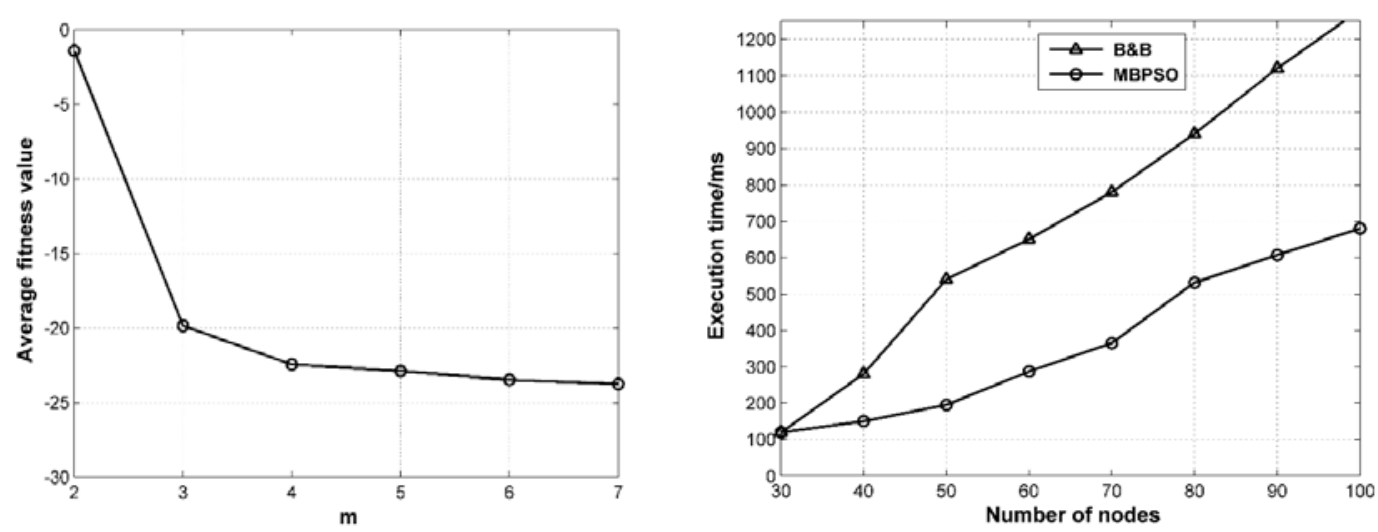
Fig.3 Performance with difference Fig.4 Algorithm execution time

Branch and Bound algorithm (B\&B) is a classic method of solving 0-1 programming problem, this paper adopted toolbox functions written by Koert Kuipers Groningen of Netherlands. Algorithm execution time as shown in Fig.5, the results show that the proposed MBPSO algorithm is obviously better than the B\&B.

\section{Summary}

A MBSPO based nodes selection algorithm was proposed to maximize measurement information for the objective function. According to the characteristics of the particles and the decision variables, we design a new particle coding method, and the V-shaped transfer function was used to improve the convergence speed. In addition, the mutation operation was used to avoid local optimum, nonlinear decreasing inertia weight to balance the global search ability and local search ability. The simulation results show that the proposed node selection mechanism can find high quality solution in a relatively short period of time.

\section{References}

[1] Akyildiz I.F, Su W, Sankarasubramaniam Y, Cayirci E. Wireless sensor network: A survey. Computer Networks, 2002, 38(4): 393-422.

[2] Chhetri, A.S,Morrell D, Papandreou-Suppappola, A. Sensor Scheduling Using a 0-1 Mixed Integer Programming Framework. Sensor Array and Multichannel Processing, Fourth IEEE Workshop on. 2006:471-475.

[3] Zoghi ,M,Kahaei ,M.H. Adaptive sensor selection in wireless sensor networks for target tracking. IET Signal Process, 2010,4(5):530-536.

[4] Kaplan, L.M. Global Node Selection for Localization in a Distributed Sensor Network. IEEE Transactions on Aerospace and Electronic Systems, 2006, 42(1):113-135.

[5] Yinfei Fu, Qing Ling, Zhi Tian . Distributed Sensor Allocation for Multi-Target Tracking in Wireless Sensor Networks. IEEE Transactions on Aerospace and Electronic Systems, 2012, 48(4):3538-3552.

[6] J.Yang,H.S.Zhang,Y.Ling and C.Peng. Task Allocation for Wireless Sensor Network Using Modified Binary Particle Swarm Optimization. IEEE SENSORS JOURNAL, 2014, 14(3):882-891.

[7] Naveed Salman, Mounir Ghogho, Andrew H. Kemp. Optimized Low Complexity Sensor Node Positioning in Wireless Sensor Networks. IEEE sensor journal.2014, 14(1):39-46.

[8] Bishopk,A.N; Jensfelt, P. An Optimality Analysis of Sensor-Target Geometries for Signal Strength Based Localization. In Proceedings of the 5th International Conference on Intelligent Sensors, Sensor Networks and Information Processing (ISSNIP), 2009:127-132. 